\title{
CAT, GPX1, MnSOD, GSTM1, GSTT1, and GSTP1 Genetic Polymorphisms in Chronic Myeloid Leukemia: A Case-Control Study
}

\author{
Claudia Bănescu, ${ }^{1}$ Adrian P. Trifa, ${ }^{2}$ Septimiu Voidăzan, ${ }^{3}$ \\ Valeriu G. Moldovan, ${ }^{1}$ Ioan Macarie, ${ }^{4}$ Erzsebeth Benedek Lazar, ${ }^{5}$ Delia Dima, ${ }^{6}$ \\ Carmen Duicu, ${ }^{7}$ and Minodora Dobreanu ${ }^{8}$ \\ ${ }^{1}$ Department of Medical Genetics, University of Medicine and Pharmacy Tirgu Mures, 38 Gh. Marinescu Street, \\ 540139 Tirgu Mures, Romania \\ ${ }^{2}$ Department of Medical Genetics, "Iuliu Hatieganu" University of Medicine and Pharmacy, 6 Louis Pasteur Street, \\ 400349 Cluj-Napoca, Romania \\ ${ }^{3}$ Department of Epidemiology, University of Medicine and Pharmacy Tirgu Mures, 38 Gh. Marinescu Street, \\ 540139 Tirgu Mures, Romania \\ ${ }^{4}$ Hematology Clinic 1, University of Medicine and Pharmacy Tirgu Mures, 50 Gh. Marinescu Street, 540136 Tirgu Mures, Romania \\ ${ }^{5}$ Hematology Clinic 2, University of Medicine and Pharmacy Tirgu Mures, 38 Gh. Marinescu Street, 540139 Tirgu Mures, Romania \\ 6 "Ion Chiricuta" Cancer Institute, 34-36 Republicii Street, 400015 Cluj-Napoca, Romania \\ ${ }^{7}$ Pediatric Clinic, University of Medicine and Pharmacy Tirgu Mures, 50 Gh. Marinescu Street, 540136 Tirgu Mures, Romania \\ ${ }^{8}$ Laboratory Medicine, University of Medicine and Pharmacy Tirgu Mures, 50 Gh. Marinescu Street, 540136 Tirgu Mures, Romania
}

Correspondence should be addressed to Claudia Bănescu; claudia.banescu@gmail.com

Received 9 August 2014; Revised 16 October 2014; Accepted 17 October 2014; Published 11 November 2014

Academic Editor: Francisco Javier Romero

Copyright (C) 2014 Claudia Bănescu et al. This is an open access article distributed under the Creative Commons Attribution License, which permits unrestricted use, distribution, and reproduction in any medium, provided the original work is properly cited.

\begin{abstract}
Oxidative damage at the DNA level may be promoted by high levels of reactive oxygen species (ROS), leading to genomic instability and increased neoplastic risk. Superoxide dismutase (SOD), glutathione peroxidase (GPX), and catalase (CAT) enzymes are implicated in the prevention of DNA damage by ROS. The aim of the study was to investigate the relationships between CAT C262T, GPX1 Pro198Leu, MnSOD Ala16Val, GSTM1, GSTT1, and GSTP1 Ile105Val polymorphisms and the risk of CML. No association was observed between CML and variant genotypes of GPX1, MnSOD, GSTM1, and GSTT1 polymorphisms in any of the investigated cases. Our study suggests that the homozygous variant genotype of the GSTP1 Ile105Val gene polymorphisms may be associated with the risk of developing $\mathrm{CML}(\mathrm{OR}=2.5 ; 95 \% \mathrm{CI}=1.08-5.7 ; P$ value $=0.02)$, while the heterozygous genotype of the $C A T$ $\mathrm{C} 262 \mathrm{~T}$ polymorphism seems to have a protective effect against $\mathrm{CML}(\mathrm{OR}=0.59,95 \% \mathrm{CI}=0.39-0.89, P$ value $=0.01)$. In most cases, no association was found between laboratory parameters and prognostic factors and the variant genotype of investigated gene polymorphisms. We concluded that CAT, GPX, MnSOD, GSTM1, and GSTT1 gene polymorphisms are not associated with the risk of CML. Variant genotype of the GSTP1 Ile105Val gene polymorphisms may contribute to the risk of developing CML.
\end{abstract}

\section{Introduction}

According to Yuzhalin and Kutikhin [1] high levels of reactive oxygen species (ROS) and long-term accumulation of ROS may promote the oxidative damage at the DNA level. This process could affect several genes responsible for the regulation of proliferation, growth, survival, apoptosis, and invasion, leading to genomic instability, deregulation of transcription, induction of mitogenic signal transduction pathways, and replication errors. All these phenomena might finally increase the risk of neoplasia [1]. Several enzymes, such as superoxide dismutase (SOD), glutathione peroxidase (GPX), catalase (CAT), nitric oxide synthase (NOS), and 
paraoxonase $(\mathrm{PON})$, function to prevent damage caused by ROS.

Manganese superoxide dismutase (MnSOD) catalyzes the conversion of highly reactive superoxide radicals to $\mathrm{H}_{2} \mathrm{O}_{2}$. Hydrogen peroxide is decomposed by mitochondrial peroxiredoxins (catalases or mitochondrial glutathione peroxidases). However, some of the polymorphisms of the MnSOD gene lead to an increased superoxide dismutase activity, yielding increased quantities of $\mathrm{H}_{2} \mathrm{O}_{2}$. This process could contribute to the neoplastic transformation of the cells [1]. Such a polymorphism of the MnSOD gene (Val16Ala), located in the mitochondrial targeting sequence, has been described to be associated with the overexpression of $M n S O D$, leading to an increased production of hydrogen peroxide $\left(\mathrm{H}_{2} \mathrm{O}_{2}\right)$, which generates increased levels of ROS, if not subsequently neutralized [2].

Catalase (CAT) participates in defense mechanisms against oxidative stress by converting $\mathrm{H}_{2} \mathrm{O}_{2}$ to water and molecular oxygen [3]. A common polymorphism of the CAT gene, namely C262T, has been associated with lower catalase activity and thus increased levels of ROS [2].

The antioxidant enzymes glutathione peroxidases (GPX) are implicated in protection of cells against oxidative damage by reducing $\mathrm{H}_{2} \mathrm{O}_{2}$ and other organic peroxidases to water with reduced glutathione [3]. A polymorphism of the GPX1 gene, namely Pro198Leu, has been shown to be associated with malignancies [4]. It was reported that this amino acid substitution decreased the enzymatic activity of glutathione peroxidase [5] in a cell line overexpressing the mutant protein compared with the same cell line overexpressing similar amounts of the wild-type enzyme. Therefore the GPX1 gene polymorphism may increase the risk of cancer induced oxidative DNA damage.

Glutathione S-transferases (GSTs) enzymes, encoded by GST genes, are implicated in detoxification of xenobiotics, and they have a crucial role in protecting tissue from oxidative damage. The variation at the GSTM1, GSTT1, and GSTP1 loci has been linked with an increase in the occurrence of cancers, probably due to an increased susceptibility to environmental toxins and carcinogens [6]. The researchers focused on several polymorphisms of the GST genes: the homozygous deletions of the GSTM1 and GSTT1 genes (the "null genotypes") and the Ile105Val substitution in the GSTP1 gene. It has been assumed that these polymorphisms lead to reduced enzymatic activity, thus decreasing their detoxification ability for certain mutagens and carcinogens. This could cause in turn DNA damage and thus a greater risk of developing cancers [7].

To our knowledge, the relationship between the CAT C262T, GPX1 Pro198Leu, and MnSOD Ala16Val gene polymorphisms and the risk of CML has not been previously investigated.

The association between GSTT1, GSTM1, and GSTP1 Ile105Val polymorphisms and the susceptibility to chronic myeloid leukemia (CML) was investigated by different studies, but with conflicting results [7-12].

The aim of the present study was to investigate the relationships between CAT C262T, GPX1 Pro198Leu, MnSOD
Ala16Val, GSTM1, GSTT1, and GSTP1 Ile105Val polymorphisms and the risk of CML.

\section{Materials and Methods}

2.1. Patients and Controls. The research protocol was approved by the Ethics Committee of the University of Medicine and Pharmacy Tirgu Mures, Romania, and written informed consent was obtained from controls and CML patients. The study was conducted in accordance with the Declaration of Helsinki.

Our study consisted of 489 individuals from the same geographical area (northwestern and central regions of Romania, comprising a population of around 6.1 million inhabitants), which were divided into two groups: the control and the patients groups.

The control group included 321 healthy individuals without a history of any type of cancer (165 females and 155 males; mean age $47.8 \pm 14.4$ years).

The patients group was composed of 168 previously untreated adult CML cases (age range $18-80$ years; mean age $51.6 \pm 13.28$ years; 71 females and 97 males) diagnosed at the Hematology Clinic from Tirgu Mures and Hematology Department of the "Ion Chiricuta" Cancer Institute from Cluj-Napoca, Romania, between December 2010 and April 2014. CML was diagnosed based on detection of Philadelphia chromosome by standard cytogenetic and/or $B C R-$ $A B L$ fusion gene by reverse transcription polymerase chain reaction.

According to the clinical phases of the disease we included our CML patients into two groups as follows: 141 cases in the group with patients in chronic phase and 27 $(16.1 \%)$ cases in the group with patients in advanced phase (accelerated and blast crisis).

Additional chromosomal abnormalities (ACA) were found in 22 (13.1\%) of our CML cases.

The frequency of ACA in CML patients in advanced phase (accelerated and blast crisis) of the disease was 59\% (13 cases).

2.2. Genotyping Procedures. Peripheral blood samples were collected in sterile tubes containing EDTA and genomic DNA was isolated using the Quick-gDNA MiniPrep kit (ZymoResearch, USA) and Wizard Genomic DNA Purification Kit (Promega, Madison, WI, USA) according to the manufacturer's instructions.

CAT C262T, GPX1 Pro198Leu, MnSOD Ala16Val, and GSTP1 Ile105Val genotyping was performed using polymerase chain reaction and restriction fragment length polymorphism (PCR-RFLP) assays as previously described [3, 13-15].

GSTM1 and GSTT1 polymorphisms were analysed using a multiplex polymerase chain reaction as previously described [16].

2.3. Statistical Analysis. Statistical analysis was performed using the Statistical Package for Social Sciences (SPSS, version 17, Chicago, IL, USA). Data were considered as nominal or quantitative variables. Nominal variables were characterized using frequencies. Quantitative variables were tested for normality of distribution using Kolmogorov-Smirnov test 
TABLE 1: Genotype distribution for the six polymorphisms investigated in patients with CML and controls.

\begin{tabular}{|c|c|c|c|c|}
\hline Polymorphism & $\begin{array}{c}\text { CML patients } \\
n(\%) \\
\end{array}$ & $\begin{array}{c}\text { Controls } \\
n(\%)\end{array}$ & $\begin{array}{c}\text { OR }(95 \% \mathrm{CI}) \\
\text { CML versus controls }\end{array}$ & $\begin{array}{c}P \text { value } \\
\text { CML versus controls } \\
\end{array}$ \\
\hline \multicolumn{5}{|l|}{ CAT C262T } \\
\hline $\mathrm{CC}$ & $105(62.5 \%)$ & $168(52.3 \%)$ & - & - \\
\hline $\mathrm{CT}$ & $49(29.2 \%)$ & $132(41.1 \%)$ & $0.59(0.39-0.89)$ & 0.01 \\
\hline $\mathrm{TT}$ & $14(8.3 \%)$ & $21(6.5 \%)$ & $1.06(0.51-2.1)$ & 0.86 \\
\hline \multicolumn{5}{|l|}{ GPX1 Pro198Leu } \\
\hline Pro/Pro & $16(9.5 \%)$ & $34(10.6 \%)$ & - & - \\
\hline Pro/Leu & $118(70.2 \%)$ & $203(63.2 \%)$ & $1.23(0.65-2.3)$ & 0.51 \\
\hline Leu/Leu & $34(20.2 \%)$ & $84(26.2 \%)$ & $0.86(0.42-1.7)$ & 0.67 \\
\hline \multicolumn{5}{|c|}{ MnSOD Ala16Val } \\
\hline Ala/Ala & $1(0.6 \%)$ & $1(0.3 \%)$ & - & - \\
\hline Ala/Val & $137(81.5 \%)$ & $253(78.8 \%)$ & $0.54(0.03-8.7)$ & 0.95 \\
\hline $\mathrm{Val} / \mathrm{Val}$ & $30(17.9 \%)$ & $67(20.9 \%)$ & $0.44(0.02-7.4)$ & 0.53 \\
\hline \multicolumn{5}{|l|}{ GSTP1 } \\
\hline Ile/Ile & $104(61.9 \%)$ & $220(68.5 \%)$ & - & - \\
\hline Ile/Val & $51(30.4 \%)$ & $90(28 \%)$ & $1.2(0.79-1.81)$ & 0.39 \\
\hline $\mathrm{Val} / \mathrm{Val}$ & $13(7.7 \%)$ & $11(3.5 \%)$ & $2.5(1.08-5.7)$ & 0.02 \\
\hline \multicolumn{5}{|l|}{ GSTM1 } \\
\hline Present & $68(40.5 \%)$ & $131(40.8 \%)$ & - & - \\
\hline Null & $100(59.5)$ & $190(59.2 \%)$ & $0.98(0.67-1.4)$ & 0.94 \\
\hline \multicolumn{5}{|l|}{ GSTT1 } \\
\hline Present & $133(79.2)$ & $67(20.9 \%)$ & - & - \\
\hline Null & $35(20.8)$ & $254(79.1 \%)$ & $0.91(0.56-1.46)$ & 0.70 \\
\hline
\end{tabular}

The bold font means statistically significant, $P<0.05$.

and were characterized by median and percentiles (25-75\%) or by mean and standard deviation (SD), when appropriate. A chi-square test was used in order to compare the frequencies of nominal variables. Quantitative variables were compared using $t$-test, Mann-Whitney test, ANOVA test, or KruskalWallis test, when appropriate. We used the Bonferroni correction or Dunn's correction in order to account for multiple comparisons. The level of statistical significance was set at $P$ value $<0.05$.

\section{Results}

The frequency of the variant CAT 262T allele in CML patients was $22.9 \%$, while in controls it was $27.1 \%(P$ value $=0.17)$. The allele frequency of the variant Leu allele for GPX1 Pro198Leu polymorphism was 50.4\% in CML group and 51.5\% in control group $(P$ value $=0.81)$. The frequency of the $M n S O D 16 \mathrm{Val}$ allele was $54.7 \%$ in the patient group and $57.8 \%$ in the control group $(P$ value $=0.43)$. The allele frequency of GSTP1 $105 \mathrm{Val}$ was $22.9 \%$ in CML patients and $17.4 \%$ in controls ( $P$ value $=0.07)$. The frequency of GSTM1 and GSTT1 null genotypes was $59.5 \%$ and $20.8 \%$, respectively, in CML group, and $59.2 \%$ and $20.9 \%$, respectively, in the control group ( $P$ value $=0.94$ and 0.99 , resp.). In addition we investigated the combined effects of GSTM1 and GSTT1 null genotypes regarding the CML risk. There were 18 patients (10.7\%) and 45 (14\%) controls harbouring both GSTM1 and GSTT1 null genotypes, which means a similar distribution $(P$ value $=0.37)$.
When analysing the genotypes of the six polymorphisms genotyped, we concluded that the variant homozygous of the GSTP1 Ile105Val polymorphism was seen significantly more frequently in patients than in controls $(\mathrm{OR}=2.5 ; 95 \% \mathrm{CI}=$ 1.08-5.7; $P$ value $=0.02$ )

Interestingly, the CAT C262T polymorphism in heterozygous state was found to be protective against $\mathrm{CML}(\mathrm{OR}=0.59$, 95\% CI $=0.39-0.89, P$ value $=0.01)$

All the genotypes for all other polymorphisms analysed shared similar frequencies between patients and controls ( $P$ value $>0.05$ for all these comparisons). The genotype distribution of the six (CAT, GPX, MnSOD, GSTP1, GSTM1, and GSTT1) gene polymorphisms in patients and controls is presented in detail in Table 1.

In addition we investigated the impact of these polymorphisms in more detail taking into account different factors such as age, gender, phase of the disease (chronic versus blast or accelerated), Hasford and Sokal risk groups, the EUTOS score, the WBC count, blasts, and basophils. No association was observed between the laboratory parameters (blast, basophils, and WBC count) and the CAT C262T, GPX1 Pro198Leu, MnSOD Ala16Val, GSTM1, GSTT1, and GSTP1 variant genotypes in CML cases.

The CAT C262T, GPX1 Pro198Leu, MnSOD Ala16Val, GSTM1, GSTT1, and GSTP1 polymorphisms investigated had no impact on the risk of CML with respect to gender $(P$ value $=0.22, P$ value $=0.31, P$ value $=0.68, P$ value $=$ $0.23, P$ value $=0.64$, and $P$ value $=0.68$, resp.) and age 
$(P$ value $=0.24, P$ value $=0.65, P$ value $=0.55, P$ value $=0.60$, $P$ value $=0.11$, and $P$ value $=0.59)$.

None of the polymorphisms GPX1 Pro198Leu, MnSOD Ala16Val, GSTM1, GSTT1, and GSTP1 was associated with Hasford and Sokal risk groups and EUTOS score in CML patients. In the case of CAT C262T polymorphism, it was not associated with Hasford risk groups and EUTOS score, but it was associated with Sokal risk group $(P$ value $=0.04)$ in $\mathrm{CML}$ patients.

We analysed the distribution of the six polymorphisms (GPX1 Pro198Leu, MnSOD Ala16Val, CAT C262T, GSTM1 and GSTT1 null genotypes, and GSTP1 Ile105Val) in patients stratified after the clinical phases of the disease (one group was represented by chronic phase and the other group was represented by accelerated or blast phase). None of the polymorphisms analyzed was associated with the clinical phases of CML ( $P$ value $>0.05$ for all comparisons performed).

Also, we performed a comparison between patients with additional chromosomal abnormalities (ACA) and variant genotypes for the investigated polymorphisms. The frequency of GSTM1 null genotype was $36.4 \%$ in CML patients with ACA and 63\% in CML patients without ACA. Our findings suggested that the GSTM1 null genotype had a protective effect against the occurrence of ACA (OR $=0.33$, 95\% CI $=0.13-0.85 ; P$ value $=0.01)$.

In the current study, no association was observed between the other five polymorphisms analyzed (GSTT1 null genotype, GSTP1 Ile105Ala, CAT C262T, GPX1 Pro198Leu, and $M n S O D$ Ala16Val) and the occurrence of ACA in CML patients ( $P$ value $>0.05$ for all comparisons performed).

\section{Discussion}

To our best knowledge, no previous studies have investigated the combined influence of CAT C262T, GPX1 Pro198Leu, MnSOD Ala16Val, GSTT1, GSTM1, and GSTP1 Ile105Val variants on CML nor the influence of these genetic polymorphisms on clinical phases of the disease and prognostic factors.

In the present study we observed no significant association between the CAT 262T, GPX1 198Leu, and MnSOD 16Val variant alleles and the risk of CML $(P$ value $=0.17, P$ value $=$ 0.81 , and $P$ value $=0.43$ ).

It has been reported that the GPX1 Pro198Leu polymorphism is associated with an increased risk of developing bladder [4, 17], breast [18], and lung [19] cancer, due to a reduction in enzymatic production and activity. No evidence regarding the association between GPX1 Pro198Leu polymorphism and prostate cancer risk [17] was found.

Taking into account that MnSOD, CAT, and GPX1 are involved in the same biological pathways, we analysed a possible synergetic effect of the polymorphisms involving the genes coding for these enzymes. The combined variant (heterozygous and homozygous) genotypes for the CAT C262T, GPX1 Pro198Leu, and MnSOD Ala16Val gene polymorphisms revealed no association with the risk of CML $(P$ value $=$ $0.17)$. The frequency of the combined variant genotypes of the CAT C262T, GPX1 Pro198Leu, and MnSOD Ala16Val polymorphisms was $33.3 \%$ (56 cases) in CML group and $40.05 \%$ (105 individuals) in control group.

In the present study, we observed that the GSTM1 and GSTT1 null genotypes were not associated with the risk of CML. These findings are in agreement with the study conducted by Löffler et al. [12] on $141 B C R-A B L$ positive CML cases and 150 random, healthy blood donors from Germany.

A positive association between GSTT1 null genotype and the risk of CML was reported by Özten et al. [11], Taspinar et al. [20], and He et al. [8]. The combined GSTM1 and GSTT1 null genotypes were significantly associated with the risk of CML $[8,11]$. Similar results were reported in a hospital-based case-control study performed on 75 CML patients and 124 unrelated volunteer controls in Kashmiri population [9].

A recent meta-analysis performed by $\mathrm{He}$ et al. [8] suggested that GSTM1 polymorphism had no effect on the susceptibility to CML.

We did not observe any association between combined GSTM1 and GSTT1 null genotypes and the risk of CML. Our findings are different compared to those reported by Bhat et al. and He et al. $[8,9]$. The conflicting results may be explained by sample size and ethnic background (the studies reported above were made in Asia).

Zhou et al. reported the results of a case-control study conducted on 163 AML cases and 204 controls and found that GSTT1 null genotype was associated with increased risk of AML in a Chinese population and no association between GSTM1 null genotype and GSTP1 Val/Val genotype and AML as independent factors [21].

According to Zhou et al., individuals bearing GSTM1, GSTT1, and GSTP1 polymorphisms have a reduced enzyme function. Therefore, they may potentially have an increased cancer susceptibility due to a decreased ability to detoxify carcinogens and reactive oxygen species [21]. Our findings revealed no association between combined GSTT1 null, GSTM1 null, and GSTP1 Val/Val genotypes and CML risk. In addition, we investigated the effects of combined GSTM1 or GSTT1 null genotype with variant GSTP1 genotypes on CML risk. We failed to find an association between variables mentioned above.

The current study indicated that the presence of homozygous variant genotype ( Val/Val) GSTP1 Ile105Val is a risk factor for CML $(\mathrm{OR}=2.5,95 \% \mathrm{CI}: 1.08-5.7, P$ value $=$ 0.02). Similar results were described in $260 \mathrm{CML}$ cases from Hyderabad, India, by Sailaja et al. [10]. On the other hand, a study from Turkey provided no evidence of a relationship between the GSTP1 IIe105Val polymorphism and susceptibility to CML [7]. Sailaja et al. suggested that CML patients in advanced phase (accelerated and blast crisis) had a higher frequency of heterozygous (Ile/Val) genotype compared to chronic phase [10]. On the contrary, we failed to find an association between variant GSTP1 Ile105Val genotypes and CML patients in accelerated and blast crisis.

The findings of different studies regarding leukemic risk and GSTT1, GSTM1, and GSTP1 lie105Val polymorphisms are presented in Table 2.

We also studied the combined genotype distribution of the investigated CAT C262T, GPX1 Pro198Leu, MnSOD Ala16Val, GSTM1, GSTT1, and GSTP1 polymorphisms in our 
TABLE 2: Findings of various studies regarding leukemic risk and GSTT1, GSTM1, and GSTP1 Ile105Val polymorphisms.

\begin{tabular}{|c|c|c|}
\hline & $\begin{array}{l}\text { Association with leukemic risk (OR, 95\% CI, } \\
P \text { value, study) }\end{array}$ & $\begin{array}{l}\text { No association with leukemic risk (OR, 95\% } \\
\text { CI, } P \text { value, study) }\end{array}$ \\
\hline \multirow{3}{*}{$\begin{array}{l}\text { Combined GSTM1 and } \\
\text { GSTT1 null genotypes }\end{array}$} & $\begin{array}{l}\text { OR }=9.47,95 \% C I=3.61-24.87, P<0.0001, \\
\text { Özten et al. }[11]\end{array}$ & \multirow{3}{*}{-} \\
\hline & $\begin{array}{l}\mathrm{OR}=1.79,95 \% \mathrm{CI}=1.24-2.58, P=0.002, \\
\text { He et al. }[8]\end{array}$ & \\
\hline & $\begin{array}{l}\mathrm{OR}=3.09,95 \% \mathrm{CI}=1.12-8.52, P=0.047 \\
\text { Bhat et al. [9] }\end{array}$ & \\
\hline \multirow{5}{*}{ GSTT1 null genotypes } & $\begin{array}{l}\mathrm{OR}=1.57,95 \% \mathrm{CI}=1.15-2.14, P=0.004, \\
\text { He et al. }[8]\end{array}$ & \multirow{5}{*}{$\begin{array}{l}\text { OR }=1.34,95 \% \mathrm{CI}=0.75-2.40, P=0.32 \text {, } \\
\text { Löffler et al. }[12]\end{array}$} \\
\hline & $\begin{array}{l}\mathrm{OR}=2.12,95 \% \mathrm{CI}=1.12-4.02 ; P=0.03 \text {, } \\
\text { Bhat et al. [9] }\end{array}$ & \\
\hline & $\begin{array}{l}\mathrm{OR}=3.53,95 \% \mathrm{CI}=2.08-6.00, P<0.0001 \text {, } \\
\text { Özten et al. }[11]\end{array}$ & \\
\hline & $\begin{array}{l}\mathrm{OR}=2.82,95 \% \mathrm{CI}=1.58-5.05, P<0.001, \\
\text { Taspinar et al. }[20]\end{array}$ & \\
\hline & $\begin{array}{l}\mathrm{OR}=1.64,95 \% \mathrm{CI}=1.03-2.63, P=0.03 \text {, } \\
\text { Zhou et al. }[21]\end{array}$ & \\
\hline \multirow{5}{*}{ GSTM1 null genotypes } & \multirow{5}{*}{-} & $\begin{array}{l}\mathrm{OR}=0.94,95 \% \mathrm{CI}=0.81-1.08, P=0.38, \\
\text { He et al. }[8]\end{array}$ \\
\hline & & $\begin{array}{l}\mathrm{OR}=1.327,95 \% \mathrm{CI}=0.73-2.40 ; P=0.4295 \text {, } \\
\text { Bhat et al. [9] }\end{array}$ \\
\hline & & $\begin{array}{l}\text { OR }=1.11,95 \% \text { CI }=0.69-1.80, P=0.714, \\
\text { Özten et al. [11] }\end{array}$ \\
\hline & & $\begin{array}{l}\text { OR }=0.92,95 \% \text { CI }=0.58-1.46, P=0.71 \text {, } \\
\text { Löffler et al. [12] }\end{array}$ \\
\hline & & $\begin{array}{l}\mathrm{OR}=1.20 ; 95 \% \mathrm{CI}=0.78-1.85 ; P=0.38 \\
\text { Zhou et al. }[21]\end{array}$ \\
\hline \multirow[b]{2}{*}{$\begin{array}{l}\text { GSTP1 Ile105Val } \\
\text { genotypes }\end{array}$} & \multirow{2}{*}{$P=0.0084$, Sailaja et al. $[10]$} & $P>0.05$, Karkucak et al. [7] \\
\hline & & $\begin{array}{l}\text { OR }=1.64 ; 95 \% C I=1.03-2.63 ; P>0.05 \\
\text { Zhou et al. }[21]\end{array}$ \\
\hline
\end{tabular}

patients with CML and controls. Our results suggest no effects of the variant combined genotypes of all six investigated gene polymorphisms and the risk of CML. Similar results were obtained in CML cases with three, four, and five variant genotypes from all the combined CAT C262T, GPX1 Pro198Leu, MnSOD Ala16Val, GSTM1, GSTT1, and GSTP1 polymorphisms.

In conclusion, our data suggests that GPX1 Pro198Leu, MnSOD Ala16Val, GSTM1, and GSTT1 gene polymorphisms are not associated with the risk of CML. Our study suggests that the homozygous variant genotype of the GSTP1 Ile105Val gene polymorphisms may contribute to the risk of developing CML, while the heterozygous genotype of the CAT C262T polymorphism seems to have a protective effect against CML.

\section{Conflict of Interests}

The authors declare no conflict of interests.

\section{Authors' Contribution}

Claudia Bănescu designed the study, performed genetic analysis, and wrote the paper. Adrian P. Trifa performed genetic analysis and revised the paper. Delia Dima, Erzsebeth
Benedek Lazar, and Ioan Macarie collected samples and data for the CML cases. Carmen Duicu and Valeriu G. Moldovan performed genetic analysis. Septimiu Voidăzan performed statistical analysis. Minodora Dobreanu designed the study and revised the paper. All authors contributed to the final version of the paper. Claudia Bănescu and Adrian P. Trifa equally contributed to this paper.

\section{Acknowledgment}

This work was supported by Internal Research Grants of the University of Medicine and Pharmacy Tirgu Mures, Romania, Project no. 19/11.12.2013.

\section{References}

[1] A. E. Yuzhalin and A. G. Kutikhin, "Inherited variations in the SOD and GPX gene families and cancer risk," Free Radical Research, vol. 46, no. 5, pp. 581-599, 2012.

[2] M. Ji, J. Tang, J. Zhao, B. Xu, J. Qin, and J. Lu, "Polymorphisms in genes involved in drug detoxification and clinical outcomes of anthracycline-based neoadjuvant chemotherapy in Chinese Han breast cancer patients," Cancer Biology and Therapy, vol. 13, no. 5, pp. 264-271, 2012. 
[3] H. S. Suzen, E. Gucyener, O. Sakalli et al., "CAT C-262T and GPX1 Pro198Leu polymorphisms in a Turkish population," Molecular Biology Reports, vol. 37, no. 1, pp. 87-92, 2010.

[4] C. Paz-y-Miño, M. J. Muñoz, A. López-Cortés et al., "Frequency of polymorphisms pro198leu in GPX-1 gene and ile58thr in MnSOD gene in the altitude ecuadorian population with bladder cancer," Oncology Research, vol. 18, no. 8, pp. 395-400, 2010.

[5] M. Bastaki, K. Huen, P. Manzanillo et al., "Genotype-activity relationship for Mn-superoxide dismutase, glutathione peroxidase 1 and catalase in humans," Pharmacogenetics and Genomics, vol. 16, no. 4, pp. 279-286, 2006.

[6] I. V. Cătană, R. A. Popp, V. P. Ioan et al., "Comparative analysis of GSTM1/GSTT1 null alleles and Ile105Val GSTP1 variant in patients with Nasal Polyposis and hyposmia in a Romanian population group," Revista Romana de Medicina de Laborator, vol. 21, no. 2, pp. 189-196, 2013.

[7] M. Karkucak, T. Yakut, T. Gulten, and R. Ali, "Investigation of GSTP1 (Ile105Val) gene polymorphism in chronic myeloid leukaemia patients," International Journal of Human Genetics, vol. 12, no. 3, pp. 145-149, 2012.

[8] H. R. He, X. X. Zhang, J. Y. Sun et al., "Glutathione S-transferase gene polymorphisms and susceptibility to chronic myeloid leukemia," Tumor Biology, vol. 35, no. 6, pp. 6119-6125, 2014.

[9] G. Bhat, A. Bhat, A. Wani et al., "Polymorphic variation in glutathione-S-transferase genes and risk of chronic myeloid leukaemia in the Kashmiri population," Asian Pacific Journal of Cancer Prevention, vol. 13, no. 1, pp. 69-73, 2012.

[10] K. Sailaja, D. Surekha, D. N. Rao, D. R. Rao, and S. Vishnupriya, "Association of the GSTP1 gene (Ile105Val) polymorphism with chronic myeloid leukemia," Asian Pacific Journal of Cancer Prevention, vol. 11, no. 2, pp. 461-464, 2010.

[11] N. Özten, A. Sunguroğlu, and M. C. Bosland, "Variations in glutathione-S-transferase genes influence risk of chronic myeloid leukemia," Hematological Oncology, vol. 30, no. 3, pp. 150-155, 2012.

[12] H. Löffler, J. Bergmann, A. Hochhaus, R. Hehlmann, and A. Krämer, "Reduced risk for chronic myelogenous leukemia in individuals with the cytochrome P-450 gene polymorphism CYP1A1*2A," Blood, vol. 98, no. 13, pp. 3874-3875, 2001.

[13] Y. Zhang, L. Zhang, D. Sun, Z. Li, L. Wang, and P. Liu, "Genetic polymorphisms of superoxide dismutases, catalase, and glutathione peroxidase in age-related cataract," Molecular Vision, vol. 17, pp. 2325-2332, 2011.

[14] M. Manica-Cattani, F. Cadoná, R. Oliveira et al., "Impact of obesity and Ala16Val MnSOD polymorphism interaction on lipid, inflammatory and oxidative blood biomarkers," Open Journal of Genetics, vol. 2, pp. 202-209, 2012.

[15] S. Hohaus, A. Di Ruscio, A. Di Febo et al., "Glutathione Stransferase P1 genotype and prognosis in Hodgkin's lymphoma," Clinical Cancer Research, vol. 11, no. 6, pp. 2175-2179, 2005.

[16] A. Sharma, A. Pandey, S. Sardana, S. Ashok, and J. K. Sharma, "Genetic polymorphisms of GSTM1 and GSTT1 genes in Delhi and comparison with other Indian and global populations," Asian Pacific Journal of Cancer Prevention, vol. 13, no. 11, pp. 5647-5652, 2012.

[17] T. Men, X. Zhang, J. Yang et al., "The rs1050450 C > T polymorphism of GPX1 is associated with the risk of bladder but not prostate cancer: evidence from a meta-analysis," Tumor Biology, vol. 35, no. 1, pp. 269-275, 2014.

[18] G. Ravn-Haren, A. Olsen, A. Tjønneland et al., "Associations between GPX1 Pro198Leu polymorphism, erythrocyte GPX activity, alcohol consumption and breast cancer risk in a prospective cohort study," Carcinogenesis, vol. 27, no. 4, pp. 820$825,2006$.

[19] D. Ratnasinghe, J. A. Tangrea, M. R. Andersen et al., "Glutathione peroxidase codon 198 polymorphism variant increases lung cancer risk," Cancer Research, vol. 60, no. 22, pp. 6381-6383, 2000.

[20] M. Taspinar, S. E. Aydos, O. Comez, A. H. Elhan, H. G. Karabulut, and A. Sunguroglu, "CYP1A1, GST gene polymorphisms and risk of chronic myeloid leukaemia," Swiss Medical Weekly, vol. 138, no. 1-2, pp. 12-17, 2008.

[21] L. Zhou, Y.-Y. Zhu, X.-D. Zhang, Y. Li, and Z.-G. Liu, "Risk effects of GST gene polymorphisms in patients with acute myeloid leukemia: a prospective study," Asian Pacific Journal of Cancer Prevention, vol. 14, no. 6, pp. 3861-3864, 2013. 


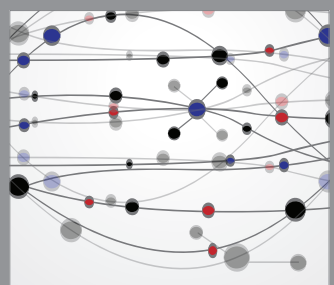

The Scientific World Journal
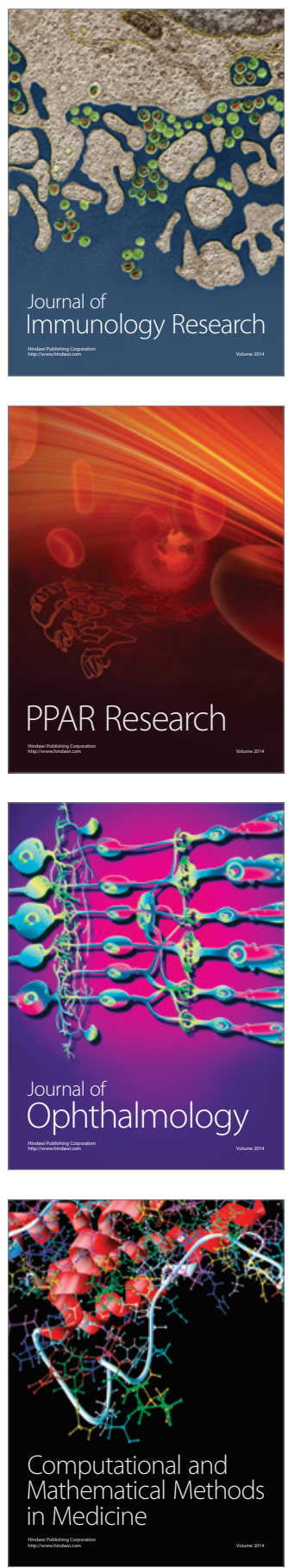

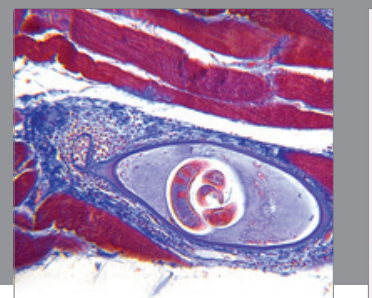

Gastroenterology

Research and Practice
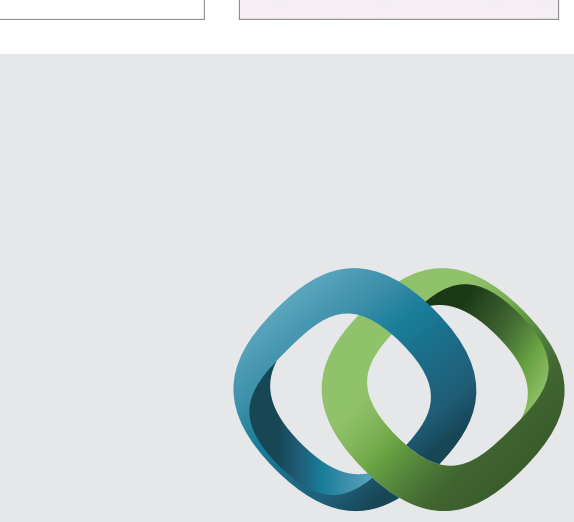

\section{Hindawi}

Submit your manuscripts at

http://www.hindawi.com
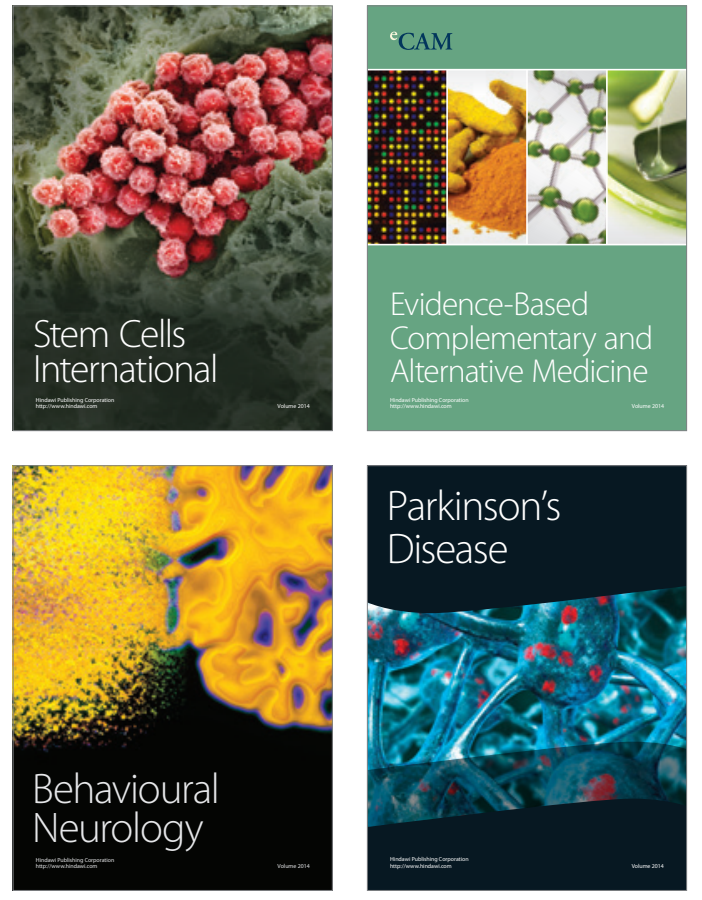
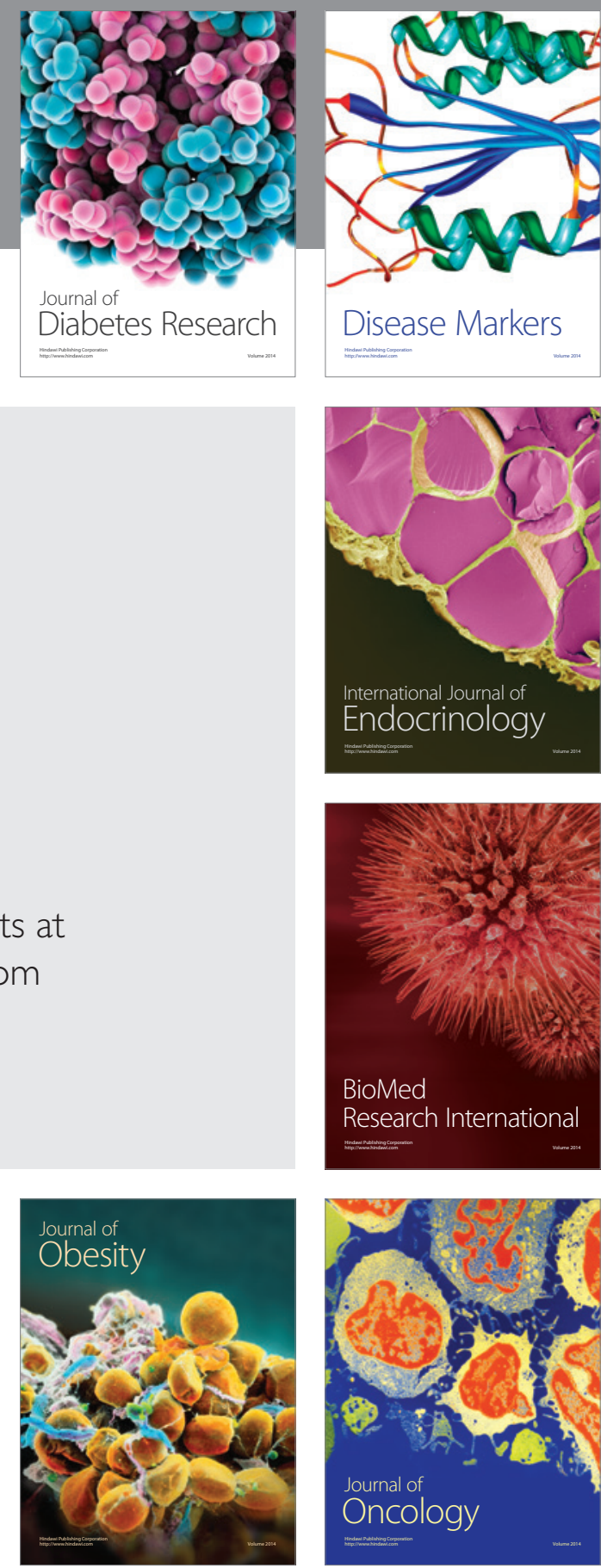

Disease Markers
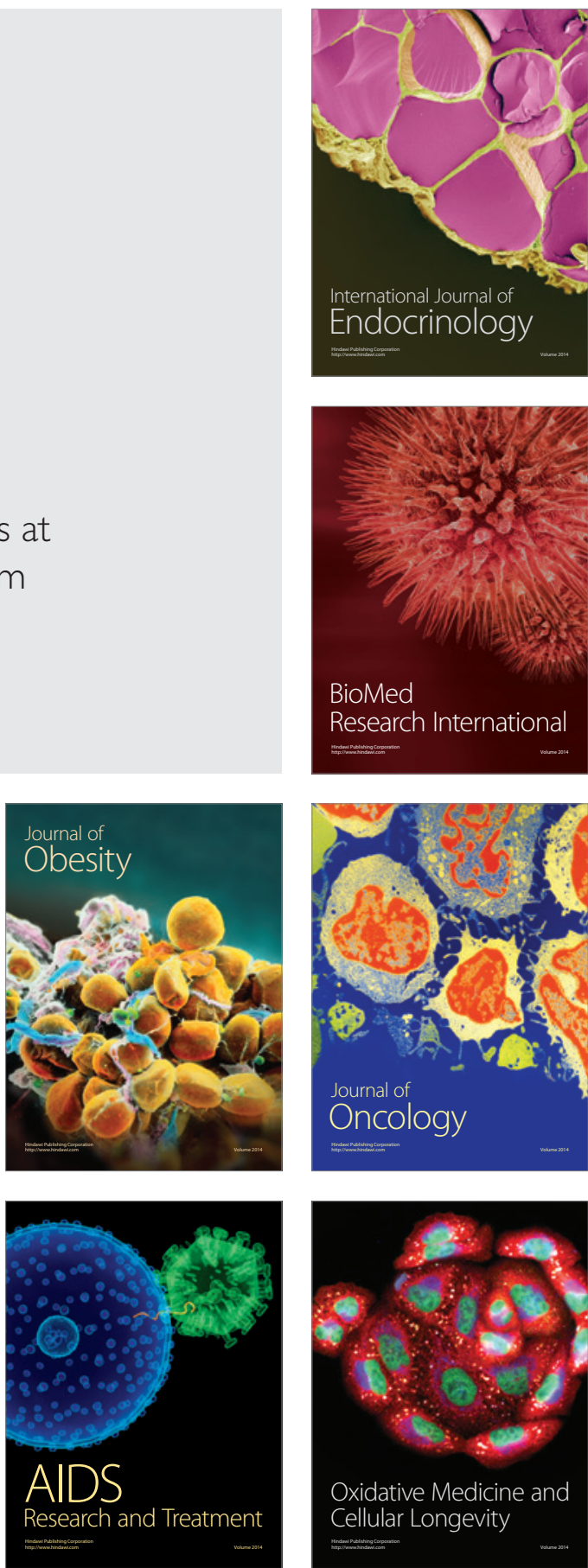